\title{
Von der sprachlichen Konstruktion des Islam
}

\author{
Nina Kalwa
}

\section{Einleitung}

Ob der Islam nach Deutschland gehört, da sind sich der derzeitige Bundespräsident Joachim Gauck und der ehemalige Bundespräsident Christian Wulff uneinig. Die Aussage Wulffs in seiner Rede zum 20. Jahrestag der deutschen Widervereinigung „Der Islam gehört zu Deutschland“ relativierte Gauck, indem er bekannte: „Ich hätte einfach gesagt, die Muslime, die hier leben, gehören zu Deutschland.“ Tatsächlich scheint es ganz intuitiv so zu sein, als stelle diese Aussage eine Relativierung der Aussage Wulffs dar. Für Gauck wie für viele andere der deutschen Gesellschaft scheint der Satz Die Muslime gehören zu Deutschland wesentlich akzeptabler als die Formulierung Der Islam gehört zu Deutschland. Der Vergleich der Reaktionen auf die beiden Äußerungen lässt darauf schließen, dass es zwischen der Bedeutung von Islam ${ }^{1}$ und der Bedeutung von Musli$m e$ fundamentale Unterschiede gibt. Dies ist vor allem deshalb erstaunlich, weil andere Äußerungen im Islamdiskurs keinen so großen Bedeutungsunterschied zwischen Islam und Muslime vermuten lassen:

(1) Der Islam begreift sich nicht nur als spirituelle Weltansicht, sondern als Weltanschauung, die das alltägliche Leben, die Politik und den Glauben als eine untrennbare Einheit sieht. ${ }^{2}$

(2) Das Welt- und Menschenbild des Islam - auch wenn es einzelne anders und demokratisch verstehen - hängt eng mit Integrationsversäumnissen zusammen. ${ }^{3}$

(3) Der Islam hat es 1.400 Jahre lang versäumt, kritische Fragen zu stellen und sich von der Politik zu lösen. ${ }^{4}$

\footnotetext{
${ }^{1}$ Im Folgenden werden alle metasprachlichen Ausdrücke kursiv gesetzt. Zudem markiere ich Bezüge auf Äußerungen im Diskurs, die keine Zitate darstellen, jedoch den Inhalt einer Äußerung wiedergeben, ebenfalls durch Kursivierung.

${ }^{2}$ FAZ (Nr. 128, S. 33), 06.06.2007, Das Minarett ist ein Herrschaftssymbol (Necla Kelek).

${ }^{3}$ SPIEGEL Online, 12.07.2007, „Wir haben wahrlich nichts zu feiern“ (Claus Christian Malzahn und Anna Reimann); http://www.spiegel.de/-politik/deutschland/-0,1518,493420,00.html.

${ }^{4}$ Ebenda.
} 
(4) Wirkliche Religionsfreiheit könne nur wechselseitig praktiziert werden. Zuvor war die Evangelische Kirche in Deutschland (EKD) dem Vorwurf muslimischer Verbände entgegengetreten, sie beabsichtige beim Dialog mit dem Islam eine christliche Mission. ${ }^{5}$

Die genannten Aussagen sind Beispiele aus dem Kölner Moscheebaudiskurs, bei dem es um den Streit um den Bau einer Moschee im Kölner Stadtteil Ehrenfeld geht. ${ }^{6}$ Wie kann der Islam als Religion sich als etwas begreifen (1), wie kann er ein bestimmtes Welt- und Menschenbild haben (2), etwas versäumen (3) oder in einen Dialog treten (4)? Die Beispiele lassen vermuten, dass Islam nicht als die „auf die im Koran niedergelegte Verkündigung des arabischen Propheten Mohammed zurückgehende Religion" verstanden wird, ${ }^{7}$ sondern dass Islam hier die Gesamtheit der Muslime bedeutet. Nicht der Islam als religiöse Lehre hat nach Auffassung der Diskursteilnehmer etwas versäumt oder hat ein bestimmtes Menschenbild, sondern die Muslime haben versäumt etc. Auch Bundeskanzlerin Angela Merkel macht in ihrer Äußerung zu der Diskussion, ob der Islam zu Deutschland gehört, deutlich, dass die religiöse Lehre letztlich nicht von ihren Anhängern zu trennen ist.

(5) Für Bundeskanzlerin Angela Merkel ist der Islam inzwischen ein fester Bestandteil Deutschlands. „Die Muslime gehören heute zu unserer Lebenswelt dazu“, sagte sie bei einem Besuch einer Schule in Berlin. Viele Muslime seien Deutsche. „Deshalb gehört der Islam eben heute dazu." ${ }^{\text {“ }}$

Weil viele Muslime Deutsche sind, gehört der Islam zu Deutschland - In dieser Schlussfolgerung Merkels wird also ein anderer Islambegriff konstituiert als in der Äußerung Gaucks. Während die Äußerung Gaucks als Reaktion auf die Aussage Wulffs annehmen lässt, dass es zwischen Islam und Muslime Bedeutungsunterschiede gibt, werden diese Bedeutungsunterschiede in der Äußerung Merkels nicht konstituiert.

\footnotetext{
${ }^{5}$ SPIEGEL Online, 10.06.2007, Kirchentagspräsident fordert Verhandlungen mit Terroristen und Taliban; http://www.spiegel.de/-panorama-/gesellschaft/-0, 1518,487704,00.html.

${ }^{6}$ Vgl. hierzu auch den Beitrag von Uwe Gerrens (S. 335-367) in diesem Sammelband.

${ }^{7}$ http://www.duden.de/rechtschreibung/Islam; zuletzt abgerufen am 30.09.2012.

${ }^{8}$ ZEIT Online, 14.05.2012, Merkel widerspricht CDU-Konservativen in Islamfrage; http://www.zeit.de/ politik/-deutschland/2012-05/merkel-islam-friedrich.
} 
Wann immer wir über den Islam reden, scheint es Konsens zu sein, was wir unter Islam verstehen. Tatsächlich verhält es sich jedoch so, dass in der Diskussion über den Islam erst ausgefochten wird, was der Islam ist. In dem vorliegenden Beitrag wird gezeigt, wie die Bedeutung von Islam, so wie sie sich im Reden über den Islam manifestiert, erfasst werden kann. Eine wichtige Prämisse dabei ist, „dass Sprache nicht als bloße Spiegelung der Wirklichkeit, sondern als ganz wesentlicher Motor ihrer Gestaltung gesehen wird". ${ }^{9}$ Das bedeutet, dass Sprache nicht nur Teil unserer Kultur ist,

sondern [sie] konstituiert diese wesentlich, und in ihr spiegeln sich nicht außersprachliche Konstellationen, Strukturen o. ä., sondern mit ihrer Analyse erhalten wir Zugang dazu, wie welche Gruppen sich mittels des sprachlichen Symbolsystems ihre Wirklichkeiten, ihr soziales Wissen erst schaffen $[\ldots]^{10}$

Wissen konstituiert und manifestiert sich in Texten. Eine Analyse der Texte erlaubt damit einen Zugriff auf dieses Wissen. Die neuere Sprachwissenschaft verfügt in ihren Unterdisziplinen der lexikalischen Semantik und der Diskurslinguistik über Methoden, die es ermöglichen, das in Texten sedimentierte, kollektive Wissen einer Gesellschaft, dabei auch das zwischen den Zeilen nur Angedeutete, aufzuzeigen. Unter einem Diskurs verstehe ich wie Andreas Gardt

die Auseinandersetzung mit einem Thema,

- die sich in Äußerungen und Texten der unterschiedlichsten Art niederschlägt,

- von mehr oder weniger großen gesellschaftlichen Gruppen getragen wird,

- das Wissen und die Einstellungen dieser Gruppen zu dem betreffenden Thema sowohl spiegelt

- als auch aktiv prägt und dadurch handlungsleitend für die zukünftige Gestaltung der gesellschaftlichen Wirklichkeit in Bezug auf dieses Thema wirkt. ${ }^{11}$

\section{Korpuslinguistik als Methode der Diskurs- und Kulturanalyse}

Diskurse sind also Orte, an denen sich Wissen konstituiert. Eine Methode, die es ermöglicht, das Wissen einer Gesellschaft aufzuzeigen, ist die Korpuslinguistik. Unter einem Korpus versteht man ganz allgemein eine Sammlung von Texten.

\footnotetext{
${ }^{9}$ Andreas Gardt 2008, S. 205.

${ }^{10}$ Martin Wengeler 2006a, S. 9.

${ }^{11}$ Andreas Gardt 2007, S. 26.
} 
Die Daten des Korpus sind typischerweise digitalisiert [...]. Die Bestandteile des Korpus, die Texte, bestehen aus den Daten sowie möglicherweise aus Metadaten, die diese Daten beschreiben, und aus linguistischen Annotationen, die diesen Daten zugeordnet sind. ${ }^{12}$

Man könnte meinen, dass die Korpuslinguistik nun die Beschäftigung mit einem beziehungsweise die Analyse eines solchen Korpus darstellt. Unter Korpuslinguistik versteht man jedoch gegenwärtig in der Sprachwissenschaft den Zugriff auf sehr große Korpora, der nicht mehr über das bloße Lesen der Texte realisiert wird, sondern mittels spezieller Computerprogramme, die beispielsweise Häufigkeiten des Vorkommens bestimmter Ausdrücke in der Umgebung anderer Ausdrücke berechnen.

Eine korpuslinguistische Analyse zum Zweck der kulturwissenschaftlichen Forschung kombiniert immer zwei verschiedene methodische Ansätze miteinander, die sogenannte corpus-driven Analyse mit der corpus-based Analyse. Während bei einer korpusbasierten (corpus-based) Untersuchung konkrete Forschungsfragen vorliegen - Beispiel: Welche Adjektive stehen im Korpus immer direkt vor Muslime? -, wird bei einer korpusgesteuerten (corpus-driven) Analyse das Korpus nicht hinsichtlich einer bestimmten Fragestellung untersucht, sondern nach allgemeinen Auffälligkeiten befragt. Beispielsweise werden bei einer sogenannten Keywordanalyse die Schlüsselausdrücke im Korpus herausgearbeitet. Welche Ausdrücke kommen im Untersuchungskorpus also signifikant häufig vor? Dies lässt sich auch auf sogenannte Mehrworteinheiten übertragen: Welche aneinander gereihten Ausdrücke sind im Korpus häufig aufzufinden? Zudem erscheint interessant, welche Ausdrücke in der nächsten Umgebung des Ausdrucks Islam vorkommen. Dies lässt sich im Rahmen einer sogenannten Kollokationsanalyse ermitteln. ${ }^{13}$ Im Rahmen dieses Beitrags werden einige der im Rahmen meines Dissertationsprojektes durchgeführten korpuslinguistischen Untersuchung dargestellt.

\footnotetext{
${ }^{12}$ Lothar Lemnitzer und Heike Zinsmeister 2006, S. 7.

${ }^{13}$ Unter einer Kollokation wird das frequente und/oder signifikante Miteinandervorkommen zweier oder mehrerer Ausdrücke in einer definierten Umgebung verstanden.
} 


\section{Das Islambild der deutschen Gesellschaft - eine korpuslinguistische Analyse}

Die Dissertation mit dem Titel „Das Konzept Islam. Eine diskurslinguistische Untersuchung" beschäftigt sich mit der Bedeutung von Islam, die sich in der öffentlichen Auseinandersetzung mit dem Islam spiegelt und gleichzeitig auch erst konstituiert wird. Um herauszufinden, was eine Diskursgemeinschaft unter Islam versteht, ist es notwendig, verschiedene Methoden miteinander zu kombinieren. Wenngleich eine korpuslinguistische Untersuchung, wie sie hier vorgestellt wird, zur vollständigen Bedeutungsbeschreibung auch nicht hinreichend sein kann ${ }^{14}$, ermöglicht sie uns einen ersten Einblick, einen ersten Hinweis darauf, was unter Islam zu verstehen ist. Geht man von der Bedeutungsdefinition Firth“ aus „You shall know a word by the company it keeps“"15, so scheint die Korpuslinguistik eine gute Methode, um Bedeutung zu erfassen, schließlich lassen sich damit eine große Menge an sprachlichen Umgebungen erfassen, in die der Ausdruck Islam eingebettet ist.

Für eine solche korpuslinguistische Untersuchung wurden drei Untersuchungskorpora erstellt. Da der 11. September 2001 unumstritten als ein wichtiges Ereignis in der Geschichte des Islamdiskurses gewertet werden kann, umfasst ein Korpus Zeitungstexte aus dem Jahr vor dem 11. September, ein weiteres Korpus Texte aus denselben Zeitungen und Magazinen aus dem Jahr nach dem 11. September. Das dritte Korpus besteht aus den jeweiligen Artikeln aus dem Jahr 2009. Somit konnte ein gegenwartsnahes Islambild der Diskursgemeinschaft mit dem Bild kurz vor und kurz nach 9/11 verglichen werden. Die Texte wurden automatisch über eine Datenbank heruntergeladen. Voraussetzung dafür, dass ein Text ins Korpus aufgenommen wurde, war die Tatsache, dass mindestens einmal die Buchstabenfolge islam oder muslim oder moslem oder moschee vorkommen musste. ${ }^{16}$

\footnotetext{
${ }^{14}$ Vgl. Nina Kalwa 2012, S. 38.

15 John Rupert Firth 1968, S. 179.

${ }^{16}$ Ein Text wurde also dann ins Korpus aufgenommen, wenn beispielsweise das freistehende Lexem Islam oder aber das Morphem islam in islamisch darin mindestens einmal vorkam.
} 
Die drei Korpora bestehen aus Artikeln aus Die WELT, Die WELT am Sonntag, Focus, taz, SPIEGEL, stern, Bonner Generalanzeiger, Berliner Morgenpost, Die Bunte und dem Hamburger Abendblatt.

\begin{tabular}{|l|l|l|}
\hline & Anzahl der Texte & Anzahl der Tokens \\
\hline Korpus „Vor 9/11“ & 3.584 Artikel & 2.086 .699 \\
\hline Korpus „Nach 9/11“ & 8.084 Artikel & 5.364 .156 \\
\hline Korpus „2009“ & 7.504 Artikel & 4.636 .483 \\
\hline
\end{tabular}

Tab. 1: Die Untersuchungskorpora

Betrachtet man die Größe der jeweiligen Korpora, so wird deutlich, wie sich das öffentliche Interesse am Gegenstand Islam im Laufe der Jahre verändert hat. Das Korpus, das die Zeitungstexte aus dem Jahr nach dem 11. September umfasst, ist mehr als doppelt so groß wie das Korpus mit den Texten aus dem Jahr vor dem 11. September. Das öffentliche Interesse am Islam ist 2009 nur unwesentlich geringer als kurz nach dem 11. September.

Im Folgenden werden einige Ergebnisse der korpuslinguistischen Untersuchung aufgezeigt, die auf das Islambild der deutschen Diskursgemeinschaft schließen lassen.

\subsection{Das Konstituieren einer islamischen Welt}

Für den Korpuslinguisten ist die Betrachtung der häufigsten Mehrworteinheiten im Korpus von besonderem Interesse. Bubenhofer spricht in diesem Zusammenhang von Sprachgebrauchsmustern. ${ }^{17}$ Diese Cluster aus mehreren Wörtern können als Musterhaftigkeiten im Diskurs betrachtet werden. Laut Foucault stellen eben diese Regelhaftigkeiten die diskursive Praxis dar. ${ }^{18}$ Die Untersuchungskorpora wurden somit auf diese Mehrworteinheiten hin untersucht. ${ }^{19}$ Bei der Durchführung der Analyse fielen besonders die häufigsten Vierworteinheiten auf.

In den unten stehenden Tabellen werden die frequentesten Vierworteinheiten gelistet, dabei wurden all jene Cluster entfernt, die sich ganz offensichtlich

\footnotetext{
${ }^{17}$ Vgl. Noah Bubenhofer 2009.

${ }^{18}$ Vgl. Michel Foucault 1981, vgl. auch Noah Bubenhofer 2009, S. 309.

${ }^{19}$ Im Folgenden verwende ich die Ausdrücke Cluster und Mehrworteinheit referenzidentisch.
} 
nicht auf die Textinhalte, sondern auf das Textlayout, d. h. die äußere Form des Textes, beziehen. Es kommen beispielsweise bestimmte Häufungen etwa von Eigennamen der jeweiligen Blätter (Axel Springer Verlag $A G$ ) vor, weil vor jedem Artikel im Korpus diese als Quelle angegeben sind. Nach der Aussortierung der Vierwort-Cluster, die sich eindeutig auf die Textoberfläche beziehen, sind nun also in den folgenden Tabellen die zehn häufigsten inhaltlichen Vierwortcluster der drei Korpora gelistet.

An den häufigsten Vierworteinheiten in den drei Korpora ist erkennbar, wie sich der Fokus auf verschiedene Teildiskurse des Islamdiskurses im Laufe der Zeit verändert hat. Es finden sich im Korpus „vor 9/11“ (Tab. 2) vor allem Einheiten, die sich auf den Nahostkonflikt beziehen, wie zwischen Israelis und Palästinensern, in Westjordanland und im und in den besetzten Gebieten. In den letzten Fahren, in der Nähe von und handelt es sich um sind Sprachgebrauchsmuster, die auf die im Korpus enthaltenen Textsorten - also Zeitungsberichte, Kommentare usw. - zurückzuführen sind. Auch die Häufigkeit von auf der anderen Seite als textstrukturierendes Element ist mit den im Korpus enthaltenen Textsorten zu begründen. ${ }^{20}$

\begin{tabular}{|l|l|}
\hline Wortgruppe & Absolute Freq \\
\hline in den letzten Jahren & 69 \\
\hline in der vergangenen Woche & 63 \\
\hline zwischen Israelis und Palästinensern & 58 \\
\hline Verbrechen gegen die Menschlichkeit & 50 \\
\hline auf der Suche nach & 45 \\
\hline in Westjordanland und im & 44 \\
\hline in den besetzten Gebieten & 44 \\
\hline handelt es sich um & 43 \\
\hline in der Nähe von & 38 \\
\hline auf der anderen Seite & 38 \\
\hline
\end{tabular}

Tab. 2: Vierwortgramme im Korpus "Vor 9/11“

\footnotetext{
${ }^{20}$ Auffällig hingegen ist auf der Suche nach, das unter den Vierworteinheiten im Korpus vor „9/11“ ebenfalls einen vorderen Rang einnimmt. Bei einer näheren Betrachtung der sprachlichen Umgebung von auf der Suche nach zeigen sich jedoch keine eindeutigen Tendenzen, deshalb soll diesem Sprachgebrauchsmuster keine weitere Beachtung geschenkt werden.
} 


\begin{tabular}{|l|l|}
\hline Wortgruppe & Absolute Freq \\
\hline New York und Washington & 577 \\
\hline nach dem \# September & 541 \\
\hline das World Trade Center & 465 \\
\hline Kampf gegen den Terrorismus & 357 \\
\hline in New York und & 338 \\
\hline auf das World Trade & 286 \\
\hline US-Präsident George W Bush & 280 \\
\hline im Kampf gegen den & 275 \\
\hline von New York und & 239 \\
\hline des World Trade Center & 220 \\
\hline
\end{tabular}

Tab. 3: Vierwortgramme im Korpus „Nach 9/11“

Wie zu erwarten war, beziehen sich die häufigsten relevanten Vierworteinheiten im Korpus „nach 9/11“ (Tab. 3) meist ganz direkt auf den Diskurs um die Anschläge vom 11. September, so nach dem \# September, das World Trade Center, im Kampf gegen den Terrorismus, auf das World Trade, des World Trade Center. Auch die anderen Vierworteinheiten in der Liste beziehen sich implizit - so wird es doch mithilfe unseres Hintergrundwissens deutlich - auf eben diesen Diskurs (New York und Washington, US-Präsident George Bush etc.). Auffällig ist, dass die Vierworteinheiten, die sich offensichtlich auf das Textthema die Anschläge des 11. Septembers beziehen, sogar noch häufiger sind als die textsortenspezifischen Wendungen aus dem Korpus „nach 9/11“, wie in den letzten fahren, in der Nähe von und auf der anderen Seite.

Bei der Liste der Vierworteinheiten im Korpus „2009“ (Tab. 4) wiederum fällt das Ergebnis heterogener aus. Einzelne Vierworteinheiten beziehen sich auf nationale, also „deutsche“ Diskurse, so etwa den Bau von Minaretten ${ }^{21}$ und der Muslime in Deutschland. Neben den textsortenspezifischen Wendungen (in den vergangenen Jahren, in den letzten fahren, auf der anderen Seite, in der Nähe von, in der vergangenen Woche) fallen zum einen US-Präsident Barack Obama und zum anderen in der islamischen Welt sowie in der arabischen Welt auf. Da Barack Obama im Januar 2009 zum 44. Präsidenten der Vereinigten Staaten von

\footnotetext{
${ }^{21}$ Genauer genommen bezieht sich den Bau von Minaretten auf einen Schweizer Volksentscheid gegen den Bau von Minaretten. Dieser Volksentscheid wurde jedoch auch in Deutschland bezüglich seiner Bedeutung für das eigene Land diskutiert.
} 
Amerika gewählt wurde und im selben Jahr eine Rede an die islamische Welt hielt, ${ }^{22}$ ist es nicht verwunderlich, dass das Vierwortcluster US-Präsident Barack Obama unter den frequentesten Clustern im Korpus „2009“ auftaucht. Auffällig hingegen ist die frequente Verwendung der Vierworteinheiten in der arabischen Welt und in der islamischen Welt.

\begin{tabular}{|l|l|}
\hline Wortgruppe & Absolute Freq \\
\hline in den vergangenen Jahren & 171 \\
\hline in der islamischen Welt & 100 \\
\hline in der arabischen Welt & 99 \\
\hline den Bau von Minaretten & 94 \\
\hline in den letzten Jahren & 80 \\
\hline auf der anderen Seite & 79 \\
\hline der Muslime in Deutschland & 79 \\
\hline von US-Präsident Barack Obama & 78 \\
\hline in der Nähe von & 76 \\
\hline in der vergangenen Woche & 76 \\
\hline
\end{tabular}

Tab. 4: Vierwortgramme im Korpus „2009“

Es stellt sich die Frage, warum die Diskursteilnehmer hier überhaupt die Vorstellung einer islamischen beziehungsweise arabischen Welt entwerfen. Wenn nun wiederum diejenigen sprachlichen Umgebungen betrachtet werden, in denen diese Cluster eingebettet sind, so fällt auf, dass die islamische Welt der westlichen Welt gegenübergestellt wird.

(6) Das westliche Demokratieverständnis ruht auf der Vorstellung des autonomen Individuums. Für die islamische Welt, meist traditionelle Gesellschaften, steht dagegen die Gemeinschaft im Zentrum, nicht der Einzelne. Familie, Klan, Sippe, Stamm und letztlich die Umma, die Gemeinschaft der Gläubigen. Die Freiheit des Individuums verwirklicht sich allein im Islam. ${ }^{23}$

(7) Die islamische Welt ist in sich tief gespalten, heterogen, von vielerlei Klüften und fremden Einflüssen geprägt. Der Unterschiede zum christlichen und nachchristlichen Europa gibt es viele, keiner prägender als die Abfolge der Revolutionen, die

\footnotetext{
${ }^{22}$ Mit Rede an die islamische Welt wurde die Rede Obamas häufig bezeichnet, so auch der Titel ihres Wikipedia-Artikels (http://de.wikipedia.org/wiki/Rede_an_die_islamische_Welt).

${ }^{23}$ SPIEGEL, 19.12.2009 (S. 102), Die Rückkehr des Allmächtigen (Marc Hujer, Andreas Lorenz, Walter Mayr, Alexander Smoltczyk, Daniel Steinvorth, Volkhard Windfuhr, Bernhard Zand).
} 
das Abendland veränderten, das Morgenland aber nicht. Das begann im Mittelalter mit dem geschichtsmächtigen Streit zwischen Kaiser und Papst. ${ }^{24}$

(8) Der Umgang mit dem Islam ist die vielleicht größte Herausforderung für Europa. Gelingt es, die eigenen Werte zu bewahren, ohne Muslime zu diskriminieren, dann kann daraus ein neuer Wertekonsens entstehen, dann könnten europäische Muslime zum Vorbild für die islamische Welt werden. Gelingt es nicht, könnte Europa seine eigenen Werte verraten, könnten die Populisten gewinnen, deren einfache Lösungen den Kampf der Kulturen anfachen. ${ }^{25}$

Die Beispiele machen deutlich, dass die islamische Welt und die westliche Welt ein Gegensatzpaar bilden. Während etwa im Westen die Vorstellung eines autonomen Individuums existiert, steht in der islamischen Welt hingegen die Gemeinschaft im Vordergrund (6). In Beispiel 7 wird explizit gesagt, dass es viele Unterschiede zwischen der westlichen und der islamischen Welt gebe. Bezogen auf die islamische Welt schwingt hier die Idee der Rückschrittlichkeit mit, wenn ausgesagt wird, dass das Abendland eine Abfolge von Revolutionen prägt, das Morgenland jedoch nicht. Schließlich wird in Beispiel 8 Europa der islamischen Welt gegenübergestellt, indem die unterschiedlichen Wertevorstellungen thematisiert werden. Insgesamt werden also die westliche und die islamische Welt als ein konträres Gegensatzpaar dargestellt: Die Zugehörigkeit zur einen Welt schließt dabei die Zugehörigkeit zur anderen Welt aus.

Was genau ist aber gemeint, wenn Diskursteilnehmer von einer islamischen Welt sprechen? Die genaue Betrachtung der sprachlichen Umgebungen dieses Clusters zeigt, dass nicht nur die Staaten, in denen die Mehrheit der Bevölkerung muslimischen Glaubens ist, zur islamischen Welt gezählt werden können. Häufig referiert der Ausdruck islamische Welt nicht auf Staaten:

(9) Ein päpstliches Zeichen gegen die „dunklen Wolken am Horizont“, gegen die drohenden Gefahren für die Menschheit durch „neue, erschütternde Konflikte“, will er setzen, einen Anstoß geben zu Kooperation und gegenseitiger Achtung aller Religionen. Wichtigster Adressat der Botschaft ist die islamische Welt. Den Anhängern des Propheten signalisierte der Papst schon vor Weihnachten mit einer symbolischen Aktion seinen Willen zu religionsüberschreitendem Respekt: Für den 14. Dezember rief er seine Gläubigen weltweit zum Fasten auf. Weil das zugleich der

\footnotetext{
${ }^{24}$ WELT (Ausg. 288, S. 7), 10.12.2009, Die Schweiz ist anders (Michael Stürmer).

${ }^{25}$ SPIEGEL, 07.12.2009 (S. 112), Angst vor Eurabien (Andrea Brandt, Marco Evers, Juliane von Mittelstaedt, Mathieu von Rohr, Britta Sandberg).
} 
letzte Tag des muslimischen Fastenmonats Ramadan war, hungerten Millionen Katholiken und Muslime gemeinsam. Viele Kardinäle in Rom, aber auch Bischöfe in den Kirchenprovinzen fanden das überhaupt nicht schicklich. ${ }^{26}$

(10) Der marokkanische Außenminister Taleb Fassi-Fihri offenbarte wenige Tage nach den Ereignissen den wahren Grund, der von Nordafrika bis in den rund 5000 Kilometer entfernten Nahen Osten Besorgnis erregt: Die islamische Welt fürchtet eine kulturelle Infiltration durch den Iran. Man habe „schiitischen Aktivismus festgestellt“, so der Außenminister, „insbesondere in der diplomatischen Vertretung in Rabat“, die sich gegen „fundamentale religiöse Werte Marokkos“ richtete und „den sunnitischen Maliki-Glauben bedrohen. ${ }^{27}$

So wird in Beispiel 9 deutlich, dass sich der Ausdruck islamische Welt nicht auf bestimmte Staaten, sondern auf die Gesamtheit aller Muslime bezieht. Die islamische Welt wird hier mittels der Nominalphrase den Anhängern des Propheten wiederaufgenommen. Referent, also die außersprachliche Bezugnahme, von islamische Welt sind also die Muslime. Auch im darauffolgenden Auszug (10) kann sich der Ausdruck nicht auf Staaten mit mehrheitlich muslimischer Bevölkerung beziehen, schließlich würde dann der Iran in diese Bezeichnung miteingeschlossen und die Äußerung die islamische Welt fürchtet eine kulturelle Infiltration durch den Iran wäre widersprüchlich. Der damalige marokkanische Außenminister Fassi-Fihri kann außerdem nicht für Staaten wie Syrien oder den Libanon sprechen. Der Referent von islamische Welt ist hier, wie in vielen anderen Beispielen, nicht ganz eindeutig. In der Äußerung in 10 kann der Ausdruck islamische Welt weder durch den Ausdruck islamische Staaten (beziehungsweise Staaten, deren Hauptreligion der Bevölkerung der Islam ist) noch durch den Ausdruck Gesamtheit der Muslime ersetzt werden.

Dass der Referent von islamische Welt oft nicht eindeutig ist, macht es umso erstaunlicher, dass der Ausdruck im Diskurs beinahe inflationär gebraucht wird. Es wird eine Rede an die islamische Welt gehalten, mit der islamischen Welt in den Dialog getreten, Politik gegenüber der islamischen Welt gemacht, es gibt Bemühungen für eine Partnerschaft mit der islamischen Welt. Wenn mit jemandem in Dialog getreten wird, Politik gegenüber jemandem gemacht wird, müsste das

\footnotetext{
${ }^{26}$ SPIEGEL, 21.01.2002 (S. 124), Christ oder Raubtier (Hans-Jürgen Schlamp).

${ }^{27}$ WELT am Sonntag (H. 14/2009, S. 8), 05.04.2009, Geheime Mission: Weltrevolution (Alfred Hackensberger).
} 
Patiens jedoch eindeutig benennbar sein. So sind im Konzept von mit jemandem in Dialog treten zwei Leerstellen offen: Man braucht jemanden, der in den Dialog tritt, also ein Agens, den aktiven Teil. Die Dialogbereitschaft geht im Untersuchungskorpus immer von einer westlichen Instanz aus: Obama tritt in den Dialog, die katholische Kirche tritt in den Dialog, die evangelische Kirche sucht den Dialog. Das Patiens, also derjenige mit dem in Dialog getreten wird, müsste eigentlich ebenfalls eindeutig benannt werden, denn ein Dialog mit einem nicht eindeutigen Referenten kann nicht funktionieren. Die vage Bezeichnung der islamischen Welt erscheint somit auffällig.

Für Hermanns ist Welt der „Totalitätsbegriff par excellence ${ }^{“ 28}$. Unter einer Totalitätsbezeichnung oder einem Totalitätsbegriff bezeichnet Hermanns all jenes Wort,

das eine Gesamtheit irgendwelcher gleichartiger Entitäten (Elemente) benennt, und zwar unabhängig davon, ob diese Gesamtheit vielleicht außerdem - aus zusätzlichen Gründen - eine „Einheit" darstellt, außer einer sozusagen rechnerischen. Eine Vorentscheidung hinsichtlich des ontologischen Charakters der Gesamtheit soll also mit der Beschreibung eines Wortes [...] als Totalitätsbezeichnung gerade nicht vorausgesetzt und impliziert bzw. suggeriert sein. Jeder neue Totalitätsbegriff konstituiert [...] einen neuen Gegenstand des Redens und Denkens, eben die „Gesamtheit“ der von ihm zusammenfassend bezeichneten Entitäten. ${ }^{29}$

Dass mit einer Totalitätsbezeichnung wie islamische Welt ein neuer Gegenstand des Redens und des Denkens konstituiert wird, konnte auch hier deutlich gemacht werden. Die islamische Welt entsteht erst im Diskurs, die Referenz des Ausdrucks ist nicht eindeutig. Laut Hermanns ist es oft sprach- und denkökonomisch

über Mengen - statt umständlich über alle ihre Elemente oder die Gesamtheit ihrer Elemente - Aussagen zu machen; deshalb (und nur deshalb) spricht man überhaupt von Mengen. Und aus diesem Vorteil läßt sich ohne weiteres wohl auch erklären, daß auch in den natürlichen Sprachen Totalitätsbezeichnungen geprägt und verwendet werden (sic!). ${ }^{30}$

Islamische Welt und arabische Welt, sogar das Wort Islam, können jeweils als Totalitätsbezeichnung im Sinne von Hermanns aufgefasst werden. Durch die

\footnotetext{
${ }^{28}$ Fritz Hermanns 1999, S. 357.

${ }^{29}$ Ebenda, S. $356 \mathrm{f}$.

${ }^{30}$ Ebenda, S. 357.
} 
Totalitätsbezeichnungen werden Einheiten sprachlich konstruiert: Eine einheitliche islamische Welt, eine arabische Welt, der Islam. Auch eine der islamischen Welt gegenüberstehende westliche Welt wird im Diskurs konstituiert. Durch die Konstitution dieses Gegensatzes entsteht die Vorstellung der Unvereinbarkeit des Islam mit der westlichen Welt, wie sie sich ebenso in der strittigen Diskussion um die Äußerungen Gaucks und Wulffs manifestiert.

\subsection{Die verschiedenen Formen des Islam}

Eine ebenfalls im Rahmen des Dissertationsprojektes durchgeführte Untersuchung des Kölner Moscheebaudiskurses ergab, dass einige Diskursteilnehmer dem Islam nicht in seiner Gesamtheit bestimmte Eigenschaften zu oder abschreiben, sondern vielmehr zwischen verschiedenen Formen des Islam unterscheiden: ${ }^{31}$

(11) Wenn man den Eindruck erweckt, der Islam - egal welcher Couleur - habe einfach keinen Platz in Deutschland, dann schweißt man die Radikalen mit den Moderaten zusammen und erzeugt so den Effekt überhaupt erst, den man anprangert. ${ }^{32}$

(12) Nordrhein-Westfalens Integrationsminister Armin Laschet (CDU) plädierte vor der Abstimmung für den Bau einer repräsentativen Moschee. Er bescheinigte der Organisation Ditib, auf dem Boden des Grundgesetzes zu stehen. Ditib stehe für einen offenen, demokratischen Islam. ${ }^{33}$

(13) Eine Fülle von Leserbriefen und Gastbeiträgen zur geplanten Kölner Zentralmoschee und zum Islam zeigt das Interesse der Bürger. Weil es um eine Moschee geht? Weil es um „den“ Islam als Religion geht? ${ }^{34}$

In Beispiel 11 ist davon die Rede, dass der Islam verschiedene Couleur haben kann. Damit macht der Sprecher deutlich, dass er nicht davon ausgeht, dass es den einen Islam gibt, sondern vielmehr verschiedene Formen des Islam unterscheidet. Ähnliches geht auch aus der Äußerung Laschets hervor (12), wenn dieser angibt, die Bauherrin der Kölner Moschee, die DİTİB, stehe für einen, offenen, demokratischen Islam. Die Anführungszeichen in Beispiel 13 zeigen, dass

\footnotetext{
${ }^{31}$ Vgl. dazu auch Nina Kalwa 2010, S. 70.

32 WELT (Heft 131/2007, S. 9), 08.06.2007, Nie wieder Hinterhof (Mariam Lau).

${ }^{33}$ SUEDEUTSCHE Online, 15.08.2007, CDU für Verkleinerung der geplanten Moschee; http://www. sued-deutsche.de/politik/koeln-cdu-fuer-verkleinerung-der-geplanten-moschee-1.239808.

${ }^{34}$ Kölner Stadtanzeiger Online, 27.08.2007, Schweigen auf rechtlichem Neuland; http://www.ksta.de/ html-/artikel/-1188192002361.shtml.
} 
auch dieser Sprecher nicht davon ausgeht, dass der Islam in Form einer Einheit existiert. Die Untersuchung machte deutlich, dass der Ausdruck Islam negative Bedeutungsanteile hervorruft. Als eine Konsequenz daraus schreiben selbst muslimische Sprecher dem Islam in seiner Gesamtheit nicht mehr bestimmte Eigenschaften zu oder ab, sondern spalten ihn in verschiedene Formen auf und weichen aus auf Formulierungen wie Wir vertreten einen moderaten Islam. ${ }^{35} \mathrm{Ob}$ dieses Phänomen nur eine Eigenart des Kölner Moscheebaudiskurses ist und ob die Diskursteilnehmer auch verschiedene Formen des Christentums und des Judentums unterscheiden, kann mithilfe einer korpuslinguistischen Analyse nachvollzogen werden. Aus diesem Grund wurde anhand des Untersuchungskorpus „2009“ zunächst überprüft, welche Wortarten jeweils direkt vor den Ausdrücken Islam, Christentum und fudentum vorkommen. Dabei fallen deutliche Unterschiede auf:

\begin{tabular}{|l|l|l|l|}
\hline Rang & Wortart & absolute Freq & relative Freq \\
\hline 1 & ART & 1.661 & 0.562288 \\
\hline 2 & APPRART & 465 & 0.157414 \\
\hline 3 & ADJA & 371 & 0.125592 \\
\hline 4 & APPR & 79 & 0.026743 \\
\hline 5 & NE & 76 & 0.025728 \\
\hline 6 & NN & 75 & 0.025389 \\
\hline 7 & KON & 73 & 0.024712 \\
\hline 8 & $\$$. & 34 & 0.011510 \\
\hline 9 & $\$$, & 31 & 0.010494 \\
\hline 10 & $\$($ & 27 & 0.009140 \\
\hline
\end{tabular}

Tab. 5: Wahrscheinlichkeit Wortart vor ,Islam' im Korpus „2009“

Insgesamt gibt es 2.954 Treffer für Islam/Islams, 318 Treffer für Christentum/Christentums und 175 Treffer für Fudentum/fudentums. Die Tabellen zeigen, welche Wortarten häufig direkt vor Islam, Christentum und Fudentum in den beiden Flexionsformen im Singular vorkommen. Die häufigste Wortart, die direkt vor einem der drei Substantive steht, ist der Artikel (ART). Bei Islam macht das im Korpus 2009 mehr als $50 \%$ aller Fälle aus. An zweiter Stelle folgt bei allen drei Substantiven - aber weitaus seltener - die Präposition mit enthaltenem

\footnotetext{
${ }^{35}$ Vgl. Nina Kalwa 2012, S. 211.
} 


\begin{tabular}{|l|l|l|l|}
\hline Rang & Wortart & absolute Freq & relative Freq \\
\hline 1 & ART & 136 & 0.427673 \\
2 & APPRART & 49 & 0.154088 \\
3 & $\$$, & 32 & 0.100629 \\
4 & KON & 31 & 0.097484 \\
5 & APPR & 25 & 0.078616 \\
6 & ADJA & 14 & 0.044025 \\
7 & $\$$. & 8 & 0.025157 \\
8 & $\$($ & 6 & 0.018868 \\
9 & ADV & 3 & 0.009434 \\
10 & CARD & 3 & 0.009434 \\
\hline
\end{tabular}

Tab. 6: Wahrscheinlichkeit Wortart vor ,Christentum' im Korpus „2009“

\begin{tabular}{|l|l|l|l|}
\hline Rang & Wortart & absolute Freq & relative Freq \\
\hline 1 & ART & 57 & 0.325714 \\
\hline 2 & APPRART & 29 & 0.165714 \\
\hline 3 & KON & 21 & 0.120000 \\
\hline 4 & APPR & 18 & 0.102857 \\
\hline 5 & $\$$, & 17 & 0.097143 \\
\hline 6 & $\$$. & 9 & 0.051429 \\
\hline 7 & ADJA & 9 & 0.051429 \\
\hline 8 & NN & 9 & 0.051429 \\
\hline 9 & $\$($ & 2 & 0.011429 \\
\hline 10 & PPOSAT & 2 & 0.011429 \\
\hline
\end{tabular}

Tab. 7: Wahrscheinlichkeit Wortart vor ,Judentum' im Korpus „2009“

Artikel (APPRART). Darunter fallen also Konstruktionen wie beim Christentum oder zum Islam. Auffällig ist jedoch, dass mit einer relativen Wahrscheinlichkeit von mehr als $12 \%$ im Korpus „2009“ vor Islam ein attributiv gebrauchtes Adjektiv (ADJA) steht. Die Wahrscheinlichkeit, dass vor Christentum ein attributiv gebrauchtes Adjektiv steht, liegt im Korpus „2009“ hingegen bei unter $5 \%$, dass vor fudentum ein solches steht bei knapp über $5 \%$. Sowohl vor Judentum als auch vor Christentum befinden sich im untersuchten Korpus häufiger eine nebenordnende Junktion (KON) oder ein Komma (\$,). Da sämtliche Texte im Korpus in irgendeiner Weise den Islam thematisieren - schließlich war dies ein Kriterium, warum die Texte überhaupt ins Korpus aufgenommen wurden -, kann man vermuten, dass diese Verteilung ihre Ursache darin hat, dass der 
Islam in einer Aufzählung mit den beiden anderen Religionen häufig zuerst genannt wird. Die Aufzählung Islam, Christentum und fudentum scheint häufiger als die Reihenfolge Christentum, fudentum und Islam. Da Islam in den Korpora insgesamt häufiger vorkommt als die beiden anderen Substantive, ist ebenfalls möglich, dass diese Aufzählungen im Falle von Islam weniger ins Gewicht fallen.

Die häufige Verwendung attributiv gebrauchter Adjektive gibt einen Hinweis darauf, dass der Islam in verschiedene Teilformen gespalten wird. Es stellt sich nun die Frage, welche attributiv gebrauchten Adjektive häufig vor Islam stehen. Zum Vergleich werden erneut auch die Adjektive vor Christentum und fudentum betrachtet:

\begin{tabular}{|l|l|l|l|}
\hline Rang & Lemma & absolute Freq & relative Freq \\
\hline 1 & politisch & 78 & 0.210243 \\
\hline 2 & $<$ unknown $>$ & 60 & 0.161725 \\
\hline 3 & radikal & 36 & 0.097035 \\
\hline 4 & schiitisch & 32 & 0.086253 \\
\hline 5 & deutsch & 16 & 0.043127 \\
\hline 6 & militant & 11 & 0.029650 \\
\hline 7 & europäisch & 9 & 0.024259 \\
\hline 8 & aufgeklärt & 8 & 0.021563 \\
\hline 9 & türkisch & 5 & 0.013477 \\
\hline 10 & wahr & 5 & 0.013477 \\
\hline
\end{tabular}

Tab. 8: Attributiv gebrauchte Adjektive vor ,Islam' im Korpus „2009“

Insgesamt kommen im Korpus nur sechs verschiedene attributiv gebrauchte Adjektive vor fudentum, 10 vor Christentum vor. Hingegen stehen im Korpus „2009“ vor Islam insgesamt 81 verschiedene attributiv gebrauchte Adjektive, von denen hier aus Darstellungsgründen nur die häufigsten zehn aufgeführt werden. ${ }^{36}$ Es wird deutlich, dass die Adjektive vor Islam einem anderen semantischen Feld zuzuordnen sind als die vor Christentum und fudentum. Politisch, radikal und militant sind Adjektive, die negative Bedeutungsaspekte hervorrufen. Das Adjektiv politisch evoziert nicht für sich genommen eine solche negative Bedeutung, sondern erst in Verbindung mit Islam, da in unserem westlichen

\footnotetext{
${ }^{36}$ Selbstverständlich muss dabei beachtet werden, dass die Ausdrücke fudentum und Christentum im Korpus insgesamt weniger frequent sind als der Ausdruck Islam.
} 


\begin{tabular}{|l|l|l|l|}
\hline Rang & Lemma & absolute Freq & relative Freq \\
\hline 1 & $<$ unknown $>$ & 3 & 0.214286 \\
\hline 2 & liberal & 2 & 0.142857 \\
\hline 3 & westlich & 2 & 0.142857 \\
\hline 4 & umfassend & 1 & 0.071429 \\
\hline 5 & aufgeklärt & 1 & 0.071429 \\
\hline 6 & orthodox & 1 & 0.071429 \\
\hline 7 & wehrhaft & 1 & 0.071429 \\
\hline 8 & friedfertig & 1 & 0.071429 \\
\hline 9 & katholisch & 1 & 0.071429 \\
\hline 10 & früh & 1 & 0.071429 \\
\hline
\end{tabular}

Tab. 9: Attributiv gebrauchte Adjektive vor ,Christentum' im Korpus „2009“

\begin{tabular}{|l|l|l|l|}
\hline Rang & Lemma & absolute Freq & relative Freq \\
\hline 1 & orthodox & 2 & 0.222222 \\
\hline 2 & modern & 2 & 0.222222 \\
\hline 3 & deutsch & 2 & 0.222222 \\
\hline 4 & ursprünglich & 1 & 0.111111 \\
\hline 5 & lebendig & 1 & 0.111111 \\
\hline 6 & traditionell & 1 & 0.111111 \\
\hline
\end{tabular}

Tab. 10: Attributiv gebrauchte Adjektive vor ,Judentum' im Korpus „2009“

Verständnis Religion und Politik unbedingt voneinander zu trennen sind. Die angebliche Eigenschaft des Politisch-Seins des Islam ruft somit eine ablehnende Haltung hervor, denn, sollte der Islam eine politische Religion sein, ist er nicht mit den westlichen Werten und dessen Demokratieverständnis zu vereinbaren.

Christentum wird mithilfe attributiv gebrauchter Adjektive hinsichtlich der verschiedenen Konfessionen näher klassifiziert (katholisch, orthodox). Liberal und friedfertig können als Adjektive betrachtet werden, die positive Bedeutung evozieren. Auch die attributiv gebrauchten Adjektive vor fudentum unterscheiden sich maßgeblich von den Adjektiven vor Islam. Alle Adjektive, die Fudentum attribuieren, sind wertneutral oder rufen positive Bedeutungsaspekte auf ( $\mathrm{mo}^{-}$ dern, lebendig).

Die Aufspaltung des Islam in verschiedene Teilformen zeigt sich also in dieser korpuslinguistischen Analyse bestätigt. Vor allem der politische Islam wird von den Diskursteilnehmern immer wieder hervorgehoben. In einem letzten Ana- 
lyseschritt wird nun untersucht, inwiefern auch die Ausdrücke Muslim beziehungsweise Muslime (oder auch Muslims) mit Adjektiven, die negative Bedeutungsaspekte evozieren, attribuiert werden:

\begin{tabular}{|l|l|l|l|}
\hline Rang & Lemma & absolute Freq & relative Freq \\
\hline 1 & jung & 77 & 0,083878 \\
\hline 2 & gläubig & 63 & 0,068627 \\
\hline 3 & $<$ unknown $>$ & 50 & 0,054466 \\
\hline 4 & deutsch & 48 & 0,052288 \\
\hline 5 & lebend & 45 & 0,04902 \\
\hline 6 & radikal & 40 & 0,043573 \\
\hline 7 & bosnisch & 31 & 0,033769 \\
\hline 8 & strenggläubig & 29 & 0,03159 \\
\hline 9 & gut & 25 & 0,027233 \\
\hline 10 & ander & 23 & 0,025054 \\
\hline
\end{tabular}

Tab. 11: Adjektive vor ,Muslim' (in den verschiedenen Flexionsformen) im Korpus „2009“

Vergleicht man nun die attributiv gebrauchten Adjektive vor Islam mit denen vor Muslim, so fällt auf, dass die Diskursteilnehmer eher dem Islam negative Eigenschaften zuschreiben als den Muslimen. Mit Ausnahme von radikal sind alle Adjektive wertneutral oder rufen positive Bedeutungsaspekte hervor $\left(g u t^{37}\right)$. Es scheint also für die Diskursteilnehmer leichter annehmbar, dem Islam negative Eigenschaften zuzuschreiben als den Muslimen. Dies führt dazu, dass der Ausdruck Islam stärkere negative Bedeutungsaspekte evoziert als der Ausdruck Muslime. Damit wird erneut deutlich, warum die Aussage Die Muslime gehören $z u$ Deutschland für viele Mitglieder der deutschen Gesellschaft weitaus akzeptabler erscheint als die Aussage Der Islam gehört zu Deutschland.

\section{Schluss}

Der Beitrag hat deutlich gemacht, dass die Bedeutung von Islam nicht feststeht, sondern sich im Reden über den Islam erst konstituiert. Während die Aussage Gaucks einen Islambegriff konstituiert, der sich stark von dem Begriff Muslime unterscheidet, suggerieren andere Aussagen, dass mit Islam letztlich doch die Muslime gemeint sind. In ihren neueren Aussagen zur Debatte begründet auch

\footnotetext{
${ }^{37}$ Gut in Verbindung mit Muslim wird im Korpus im Sinne von gläubig verwendet.
} 
Bundeskanzlerin Merkel ihre Annahme, dass der Islam „ein Teil von uns“ ist damit, „dass mittlerweile mehr als drei Millionen Muslime in Deutschland leben. ${ }^{\text {“3 }}$ Damit wird in den Aussagen Merkels, der Gegensatz von WIR und DIE, der in vielen anderen Aussagen im Diskurs konstituiert wird, aufgehoben. Weil der Islam beziehungsweise die islamische Welt im Diskurs häufig der westlichen Welt gegenübergesetzt wird, weil sich das Reden von einem radikalen, politischen Islam häuft, evoziert der Ausdruck Islam negative Bedeutungsanteile - so macht es die Betrachtung seiner sprachlichen Umgebungen im Korpus deutlich. Dies ist einer der Gründe, warum viele Deutsche den Satz Die Muslime gehören zu Deutschland eher bejahen als die Aussage Der Islam gehört zu Deutschland.

\section{Literatur}

Barsch, Achim; Scheuer, Helmut und Schulz, Georg-Michael (Hrsg.) (2008), Literatur Kunst - Medien. Festschrift für Peter Seibert zum 60. Geburtstag. München.

Bubenhofer, Noah (2009), Sprachgebrauchsmuster. Korpuslinguistik als Methode der Diskurs- und Kulturanalyse. Berlin.

Firth, John Rupert (1968), “A synopsis of Linguistic Theory”. In: Palmer (Hrsg.), Selected papers of J.R. Firth 1952-1959, 168-205.

Foucault, Michel (1981), Archäologie des Wissens. Frankfurt a. M.

Gardt, Andreas; Haß-Zumkehr, Ulrike und Roelcke, Thorsten (Hrsg.) (1999), Sprachgeschichte als Kulturgeschichte. Berlin/New York (Studia Linguistica Germanica, Bd. 54).

Gardt, Andreas (2007), Diskursanalyse. Aktueller theoretischer Ort und methodische Möglichkeiten. In: Warnke (Hrsg.), Diskurslinguistik nach Foucault, 28-52.

Gardt, Andreas (2008), Kunst und Sprache. Beobachtungen anlässlich der documenta 12. In: Barsch, Scheuer und Schulz (Hrsg.), Literatur - Kunst - Medien, 201-224.

Hermanns, Fritz (1999), Sprache, Kultur und Identität. Reflexionen über drei Totalitätsbegriffe. In: Gardt, Haß-Zumkehr und Roelcke (Hrsg.), Sprachgeschichte als Kulturgeschichte, 351-391.

Kalwa, Nina (2010), „Islam“-Konzepte im Kölner Moscheebaudiskurs: Eine frame-semantische Analyse zum Islambegriff. In: Aptum 1/2010, S. 55-75.

Kalwa, Nina (2012), Das Konzept Islam. Eine diskurslinguistische Untersuchung. (Diss. Universität Kassel).

Lemnitzer, Lothar und Zinsmeister, Heike (2006), Korpuslinguistik. Eine Einführung. Tübingen.

${ }^{38}$ WELT Online, 26.09.2012, Merkel - „Der Islam ist ein Teil von uns“; http://www.welt.de/politik/ deutschland-/article109489800/Merkel-Der-Islam-ist-ein-Teil-von-uns.html. 
Palmer, Frank Robert (Hrsg.) (1968), Selected papers of J. R. Firth 1952-1959. London.

Schmid, Helmut (1994), Probabilistic part-of-speech tagging using decision trees. In: Proceedings of the International Conference on New Methods in Language Processing. Manchester, UK. (Online abrufbar als PDF unter ftp://ftp.ims.uni-stuttgart.de/pub/ corpora/tree-tagger1.pdf; zuletzt abgerufen am 30.09.2012.)

Warnke, Ingo H. (Hrsg.) (2007), Diskurslinguistik nach Foucault. Theorie und Gegenstände. Berlin.

Wengeler, Martin (2006a), Linguistik als Kulturwissenschaft. Eine Einführung in diesen Band. In: Wengeler (Hrsg.), Linguistik als Kulturwissenschaft, 1-23.

Wengeler, Martin (Hrsg.) (2006b), Linguistik als Kulturwissenschaft. Hildesheim/New York. 